\title{
Estimating bias in causes of death ascertainment in the Finnish Randomized Study of Screening for Prostate Cancer
}

\section{Kilpeläinen, Tuomas $P$.}

2016-12

Kilpeläinen , T P , Makinen , T , Karhunen , P J , Aro , J , Lahtela , J , Taari , K, Talala , K, Tammela , T L J \& Auvinen, A 2016 , ' Estimating bias in causes of death ascertainment in the Finnish Randomized Study of Screening for Prostate Cancer ' , Cancer Epidemiology , vol. 45 , pp. 1-5 . https://doi.org/10.1016/j.canep.2016.08.022

http://hdl.handle.net/10138/230309

https://doi.org/10.1016/j.canep.2016.08.022

publishedVersion

Downloaded from Helda, University of Helsinki institutional repository.

This is an electronic reprint of the original article.

This reprint may differ from the original in pagination and typographic detail.

Please cite the original version. 


\title{
Estimating bias in causes of death ascertainment in the Finnish Randomized Study of Screening for Prostate Cancer
}

\author{
Tuomas P. Kilpeläinen, M.D., Ph.D. ${ }^{a, *}$, Tuukka Mäkinen, M.D., Ph.D. ${ }^{\text {b }}$, \\ Pekka J. Karhunen, professor ${ }^{\mathrm{c}}$, Jussi Aro, M.D., Ph.D. ${ }^{\mathrm{a}}$, Jorma Lahtela, professor ${ }^{\mathrm{d}}$, \\ Kimmo Taari, professor ${ }^{\mathrm{a}}$, Kirsi Talala, Ph.D. ${ }^{\mathrm{e}}$, Teuvo L.J. Tammela, professor ${ }^{\mathrm{f}}$, \\ Anssi Auvinen, professor ${ }^{\mathrm{g}}$ \\ a Dept. of Urology, University of Helsinki and Helsinki University Hospital, FI-00029 Helsinki, Finland \\ ${ }^{\mathrm{b}}$ Department of Surgery, Seinäjoki Central Hospital, FI-60220 Seinäjoki, Finland \\ ${ }^{\mathrm{c}}$ Department of Forensic Medicine, School of Medicine, University of Tampere and Fimlab Laboratories, Tampere University Hospital Region, Finland \\ ${ }^{\mathrm{d}}$ Department of Internal Medicine, Tampere University Hospital, FI-33521 Tampere, Finland \\ e Finnish Cancer Registry, FI-00130 Helsinki, Finland \\ ${ }^{\mathrm{f}}$ Dept. of Urology, University of Tampere and Tampere University Hospital, FI-33521 Tampere, Finland \\ ${ }^{g}$ School of Health Sciences, University of Tampere, FI-33014 Tampere, Finland
}

\section{A R T I C L E I N F O}

\section{Article history:}

Received 24 May 2016

Received in revised form 23 August 2016 Accepted 30 August 2016

Available online 14 September 2016

Keywords:

Mass screening

Prostatic neoplasms

Prostate-specific antigen

Randomized controlled trials

Mortality

Cause of death

\begin{abstract}
A B S T R A C T
Background: Precise cause of death (CoD) ascertainment is crucial in any cancer screening trial to avoid bias from misclassification due to excessive recording of diagnosed cancer as a CoD in death certificates instead of non-cancer disease that actually caused death. We estimated whether there was bias in CoD determination between screening (SA) and control arms (CA) in a population-based prostate cancer (PCa) screening trial.

Methods: Our trial is the largest component of the European Randomized Study of Screening for Prostate Cancer with more than 80,000 men. Randomly selected deaths in men with PCa ( N $=442 / 2568$ cases, $17.2 \%$ ) were reviewed by an independent $\mathrm{CoD}$ committee. Median follow-up was 16.8 years in both arms. Results: Overdiagnosis of PCa was present in the SA as the risk ratio for PCa incidence was 1.19 (95\% confidence interval (CI) 1.14-1.24). The hazard ratio (HR) for PCa mortality was $0.94(95 \% \mathrm{CI} 0.82-1.08)$ in favor of the SA. Agreement with official CoD registry was $94.6 \%(\kappa=0.88)$ in the SA and $95.4 \%(\kappa=0.91)$ in the CA. Altogether 14 PCa deaths were estimated as false-positive in both arms and exclusion of these resulted in HR 0.92 (95\% CI 0.80-1.06).

Conclusions: A small differential misclassification bias in ascertainment of CoD was present, most likely due to attribution bias (overdiagnosis in the SA). Maximum precision in CoD ascertainment can only be achieved with independent review of all deaths in the diseased population. However, this is cumbersome and expensive and may provide little benefit compared to random sampling.
\end{abstract}

(c) 2016 Elsevier Ltd. All rights reserved.

\section{Introduction}

Population-based prostate cancer (PCa) screening remains controversial despite evidence for mortality reduction by the European Randomized Study of Screening for Prostate Cancer (ERSPC) [1]. The problem with overdiagnosis of low-risk PCas and

\footnotetext{
Abbreviations: CoD, cause of death; SA, screening arm; CA, control arm; PCa, prostate cancer; $\mathrm{CI}$, confidence interval; HR, hazard ratio; ERSPC, European Randomized Study of Screening for Prostate Cancer; PLCO, prostate, lung, colorectal and ovarian cancer screening trial; PSA, prostate-specific antigen.

* Corresponding author.

E-mail address: tuomas.kilpelainen@hus.fi (T.P. Kilpeläinen).
}

subsequent overtreatment as well as issues of cost-effectiveness and quality of life remain to be fully evaluated [2-5].

The Finnish Randomized Study of Screening for Prostate Cancer is the largest component of the multinational ERSPC trial with over 80,000 men. The population-based Finnish trial showed a nonsignificant $15 \%$ relative reduction in PCa mortality at 12 years of follow-up (HR 0.85; 95\% confidence interval (CI) 0.69-1.04) [6]. In a subsequent analysis, non-participation in the screening arm (SA) was shown to have a major impact on PCa mortality in the Finnish trial, as correcting for non-participation improved the result into HR 0.78 (95\% CI 0.64-0.96) [7]. A substantial diluting effect may also be caused by screening contamination (i.e. unorganized 
prostate-specific antigen (PSA) testing) in the control arm (CA), which remains to be addressed in detail.

A cornerstone in any mortality study is accuracy of data on causes of death (CoD), and therefore it is customary to establish an independent $\mathrm{CoD}$ review committee to validate these data. Such review committees have been used both in the ERSPC trial [8] and Prostate, Lung, Colorectal and Ovarian (PLCO) trial [9]. The Finnish trial also used a CoD committee that systematically reviewed all deaths in patients with diagnosed PCa in 1996-2003, blinded in terms of death certificate and trial arm. The agreement between official causes of death and the committee was shown to be excellent (97.7\%; $\kappa=0.95$ ) [10] suggesting reliability of official CoD statistics in Finland and therefore systematic review was discontinued. Official $\mathrm{CoD}$ data have since been used in mortality estimates, because reviewing individually all deaths in men with PCa would be far too laborious, given little improvement over registered CoD.

The purpose of this study was to analyze whether there was bias present in CoD ascertainment by trial arm, i.e. differential misclassification that could affect the mortality results. A secondary purpose was to reassess the level of agreement between official CoD registry and the random cases reviewed by the CoD committee.

\section{Materials \& methods}

The Finnish Randomized Study of Screening for Prostate Cancer is the largest single center of the European Randomized Study of Screening for Prostate Cancer, with altogether 31868 (39.8\%) men in the screening arm and 48299 (60.2\%) men in the control arm. The ERSPC trial is registered (http://registered-trials.com, number ISRCTN49127736). The trial protocol has been published in detail previously [6]. To summarize, the men born in 1929-1944 (aged 55-67 years at entry) were identified from the population registry. A random sample of 8000 men was annually allocated to the SA and the rest of the men in each age group formed the CA. The men in the CA were not contacted.

The men in the SA were invited to a local clinic for determination of serum PSA. Men with a PSA $\geq 4.0 \mathrm{ng} / \mathrm{ml}$ were referred to a urological clinic for diagnostic examinations including digital rectal examination, transrectal ultrasound and prostate biopsy. Men with PSA of $3.0-3.99 \mathrm{ng} / \mathrm{ml}$ were referred to an additional test, which in 1996-1998 was digital rectal examination and since 1999 determination of the free/total PSA ratio with a cutoff of $16 \%$. Men with an abnormal additional test were referred for diagnostic examinations, similar to those with PSA $\geq 4.0 \mathrm{ng} / \mathrm{ml}$.

The men were re-invited to the screening test four and eight years after the first screen. Information on PCas detected outside the screening protocol and in the CA were obtained from the Finnish Cancer Registry, which has a nearly complete coverage (99\%) of solid cancers diagnosed in Finland [11].

The follow-up ended at death, emigration from Finland or common closing date, which in this study was December 31st, 2014. All deaths in Finland are registered by Statistics Finland, and the current 10th revision of the International Classification of Diseases (ICD-10) has been used since 1996.

To validate the quality of official CoD registry, in 1996-2003 all deaths in men with PCa diagnosis (regardless of the trial arm) were evaluated by a CoD committee with three members (specialists in the fields of urology, forensic pathology and internal medicine). The members had access to patient records, imaging studies and medical charts from both hospital records and outpatient clinics. The members evaluated the reports independently and were blinded from the official death certificate information, patient identity, trial arm and method of cancer detection. In cases of disagreement between individual reviewers, a consensus was sought in joint meetings of the committee.

A flowchart was utilized to estimate the role of underlying PCa in causing death [8]. The World Health Organization defines the underlying $\mathrm{CoD}$ as the disease or external injury that initiated the process that led to death. Treatment complications due to management of PCa were classified as PCa deaths according to the World Health Organization definition. Altogether 179 cases of 442 underwent autopsy.

PCa mortality and all-cause mortality were estimated using the Cox proportional hazards regression and Schoenfeld residuals were used to verify the proportionality assumption. PCa incidence between trial arms was estimated using Poisson regression with person-years (the proportionality assumption was violated with Cox regression in regard to $\mathrm{PCa}$ incidence).

The CoD committee was considered the gold standard. Sensitivity for identifying a PCa death was estimated as the proportion of correctly identified PCa deaths (concordance of official causes with the committee assessment) divided by all PCa deaths (according to the $\mathrm{CoD}$ committee). Specificity was calculated as true negatives (non-PCa deaths estimated by official registry) divided by all those that were non-PCa deaths (based on the CoD committee review). Positive predictive value was estimated as the probability that a case labeled as PCa death by the official registry was indeed correct and negative predictive value as the probability that a non-PCa death classified by the official registry was correct (Table 1 ). Agreement was estimated by Cohen's $\kappa$ statistic [12]. The correcting factor was calculated in both trial arms separately. The correcting factor was estimated by dividing the number of actual PCa deaths identified by the CoD committee by the number of PCa deaths identified by official CoD registry (Table 1 ). The number of PCa deaths in the SA and CA were multiplied by this correcting factor to estimate the amount of true number of PCa deaths in each arm.

Stata 10 (StataCorp, CollegeStation, TX, USA) was used for all analyses. 95\% confidence intervals were used and all statistical tests were two-sided.

The study protocol was approved by Helsinki and Tampere University Hospital Ethics committees. Permission to use cancer registry data was obtained from Research and Development Centre for Welfare and Health (STAKES, currently National Institute for Health and Welfare).

\section{Results}

There were altogether 31868 men in the SA and 48299 men in the CA (Fig. 1). The median follow-up time was 16.8 years in both arms. A total of 3587 PCas were diagnosed in the SA (cumulative incidence $11.3 \%$ ) and $4684(9.7 \%$ ) in the CA (RR 1.19 with $95 \%$ CI $1.14-1.24 ; \mathrm{p}<0.0001)$.

At the end of follow-up, 319 (1.0\%) men had died of PCa in the SA and 517 (1.1\%) in the CA (PCa mortality HR 0.94 with 95\% CI 0.82$1.08, \mathrm{p}=0.375)$. Altogether $10605(33.3 \%)$ men had died of other causes in the SA and 15989 (33.1\%) in the CA (all-cause mortality HR 1.01 with $95 \%$ CI $0.98-1.03 ; \mathrm{p}=0.615)$.

In the SA, there were 1115 (3.5\%) men who were diagnosed with PCa and died of any cause during the follow-up and in the CA altogether 1453 (3.0\%) such men. Of these deaths, the CoD committee evaluated 205 (in the SA) and 237 (in the CA) (Table 1).

Overall, the agreement between the CoD committee and official death certificates was $95.0 \%$. Based on the cases reviewed by the CoD committee, there were altogether 7 non-PCa deaths that were mislabeled by the official records as PCa deaths in the SA (7) $68=10.3 \%$ ) and conversely 4 incorrectly classified non-PCa deaths $(4 / 137=2.9 \%)$ that turned out to be PCa deaths (Table 1$)$. Therefore, it is estimated that with a correcting factor of 65/68 there should be 
Table 1

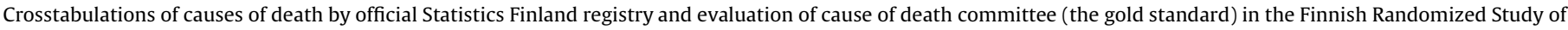
Screening for Prostate Cancer (1996-2014).

\begin{tabular}{|c|c|c|c|}
\hline \multicolumn{4}{|l|}{ Screening arm } \\
\hline \multirow[t]{2}{*}{ Statistics Finland } & \multicolumn{3}{|c|}{ Cause of death committee } \\
\hline & Prostate cancer death & Other cause of death & \\
\hline Prostate cancer death & 61 & 7 & 68 \\
\hline \multirow[t]{2}{*}{ Other cause of death } & 4 & 133 & 137 \\
\hline & 65 & 140 & 205 \\
\hline Sensitivity $(=61 /(61+4))$ & & $0.94(95 \%$ CI $0.84-0.98)$ & \\
\hline Specificity $(=133 /(133+7))$ & & 0.95 (95\% CI $0.90-0.98)$ & \\
\hline Positive predictive value $(=61 /(61+7))$ & & 0.90 (95\% CI 0.79-0.95) & \\
\hline Negative predictive value $(=133 /(133+4))$ & & 0.97 (95\% CI 0.92-0.99) & \\
\hline Agreement & & $94.6 \%$ & \\
\hline Expected agreement & & $56.2 \%$ & \\
\hline Kappa & & 0.88 (95\% CI $0.82-0.94)$ & \\
\hline Correcting factor $(=65 / 68)$ & & 0.956 & \\
\hline \multicolumn{4}{|l|}{ Control arm } \\
\hline \multirow[t]{2}{*}{ Statistics Finland } & \multicolumn{3}{|c|}{ Cause of death committee } \\
\hline & Prostate cancer death & Other cause of death & \\
\hline Prostate cancer death & 105 & 7 & 112 \\
\hline \multirow[t]{2}{*}{ Other cause of death } & 4 & 121 & 125 \\
\hline & 109 & 128 & 237 \\
\hline Sensitivity $(=105 /(105+4))$ & & 0.96 (95\% CI 0.90-0.99) & \\
\hline Specificity $(=121 /(121+7))$ & & 0.95 (95\% CI 0.89-0.98) & \\
\hline Positive predictive value $(=105 /(105+7))$ & & $0.94(95 \%$ CI $0.87-0.97)$ & \\
\hline Negative predictive value $(=121 /(121+4))$ & & 0.97 (95\% CI 0.92-0.99) & \\
\hline Agreement & & $95.4 \%$ & \\
\hline Expected agreement & & $50.2 \%$ & \\
\hline Kappa & & 0.91 (95\% CI 0.86-0.95) & \\
\hline Correcting factor $(=109 / 112)$ & & 0.973 & \\
\hline
\end{tabular}

$319^{*}(65 / 68)=305$ PCa deaths in the SA (i.e. altogether 14 fewer PCa deaths). Similarly, in the CA, in official statistics there were 7 nonPCa deaths that had been misclassified PCa deaths $(7 / 112=6.3 \%)$ and 4 PCa deaths that had been misclassified non-PCa deaths (4/ $125=3.2 \%)$; thus there should be $517^{*}(109 / 112)=503$ PCa deaths in the CA (i.e. altogether 14 fewer PCa deaths). When the presumably false positive PCa deaths $(\mathrm{N}=14)$ were excluded from the SA and from the $\mathrm{CA}(\mathrm{N}=14)$, the resulting $\mathrm{HR}$ was 0.92 (95\% $\mathrm{CI} 0.80-1.06$; $\mathrm{p}=0.267)$.

Inter-observer agreement was $94.6 \%$ in the SA $(\kappa=0.88)$ and $95.4 \%$ in the CA $(\kappa=0.91)$. There were no essential differences in sensitivity, specificity, positive predictive value or negative predictive value between the trial arms (Table 1 ).
The most common CoD in the trial were diseases of the circulatory system and cancer (Table 2). The CoD distribution was equal between trial arms and no statistically significant differences were observed (results not shown). Altogether four cases were identified as intervention-related PCa deaths, and two of these were missed by death certificates (both were from the SA).

\section{Discussion}

In any trial with disease-specific mortality as an endpoint, causes of death must be determined as precisely as possible to avoid bias. Particular concern is differential misclassification, which means that the mortality results are not comparable

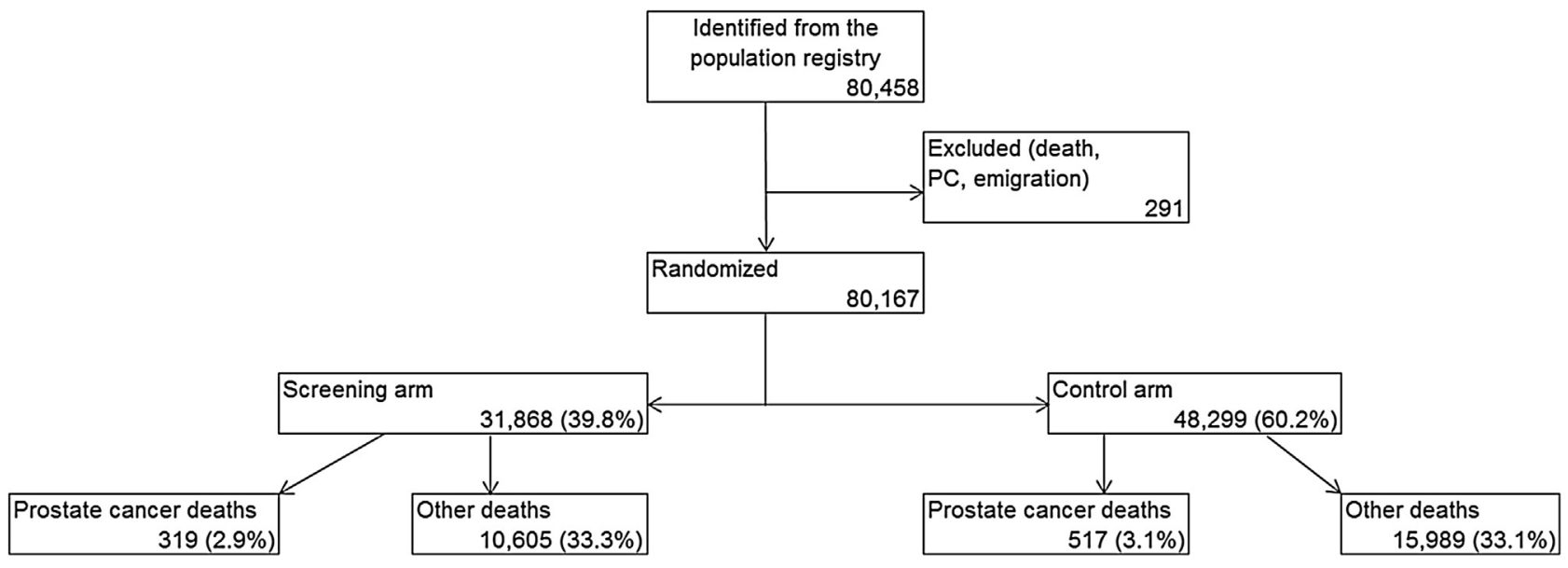

Fig. 1. A CONSORT-style flow chart of the Finnish Randomized Study of Screening for Prostate Cancer (1996-2014). 
Table 2

Cause of death distribution in the Finnish Randomized Study of Screening for Prostate cancer (1996-2014) according to ICD-10 classification.

\begin{tabular}{|c|c|c|c|}
\hline Cause of death & $\begin{array}{l}\text { Control arm } \\
\mathrm{N}(\%)\end{array}$ & $\begin{array}{l}\text { Screening arm } \\
\mathrm{N}(\%)\end{array}$ & Total \\
\hline Infectious diseases (A00-B99) & $135(0.8)$ & $82(0.8)$ & 217 \\
\hline Prostate cancer (C61) & $517(3.1)$ & $319(2.9)$ & 836 \\
\hline Other urological cancers (C60-68, not C61) & $317(1.9)$ & $202(1.8)$ & 519 \\
\hline Other cancers (C00-C96, not C60-C68) & $4163(25.2)$ & $2755(25.2)$ & 6918 \\
\hline Benign tumours (D10-36) & $8(0.0)$ & $11(0.1)$ & 19 \\
\hline Unknown tumours (D37-48) & $57(0.3)$ & $44(0.4)$ & 101 \\
\hline Diseases of the blood and blood-forming organs (D50-89) & $18(0.1)$ & $6(0.1)$ & 24 \\
\hline Endocrine, nutritional and metabolic diseases (E00-E90) & $212(1.3)$ & $142(1.3)$ & 354 \\
\hline Mental and behavioural disorders (F00-F99) & $339(2.1)$ & $219(2.0)$ & 558 \\
\hline Diseases of the nervous system (G00-G99) & $980(5.9)$ & $685(6.3)$ & 1665 \\
\hline Diseases of the circulatory system (I00-I99) & $6456(39.1)$ & $4208(38.5)$ & 10,664 \\
\hline Diseases of the respiratory system (J00-J99) & $1001(6.1)$ & $719(6.6)$ & 1720 \\
\hline Diseases of the digestive system (K00-K93) & $873(5.3)$ & $583(5.3)$ & 1456 \\
\hline Diseases of the skin (L00-L99) & $12(0.1)$ & $4(0.0)$ & 16 \\
\hline Diseases of the musculoskeletal system (M00-M99) & $51(0.3)$ & $33(0.3)$ & 84 \\
\hline Diseases of the genitourinary system (N00-N99) & $68(0.4)$ & $46(0.4)$ & 114 \\
\hline Congenital illnesses and abnormalities (Q00-Q99) & $16(0.1)$ & $9(0.1)$ & 25 \\
\hline Symptoms and signs of abnormalilies (R00-R99) & $111(0.7)$ & $72(0.7)$ & 183 \\
\hline \multirow[t]{2}{*}{ Injury, poisoning or other external cause (S00-T98) } & $1172(7.1)$ & $785(7.2)$ & 1957 \\
\hline & $16,506(100.0)$ & $10,924(100.0)$ & \\
\hline
\end{tabular}

between the trial arms, resulting in a biased estimate of effect. This study shows that some differential bias was present in the Finnish Randomized Study of Screening for Prostate Cancer, but this is unlikely to cause substantial error in PCa mortality. At a median 16.8 years of follow-up, the uncorrected PCa mortality HR was 0.94 (95\% CI 0.82-1.08) in favor of screening and with CoD correction, the HR improved to $0.92(0.80-1.06)$.

Biases that can cause differential misclassification in cancer screening trials have been divided into two types [13]. Attribution bias (so-called "sticky diagnosis" bias) is caused by the fact that in the intervention (screening) arm more cancers are diagnosed and subsequently it is more probable that deaths in the screened population are attributed to the particular cancer than in the control population, as a previous diagnosis may be entered into the death certificate even if this disease does not initiate the chain of events that causes death. Attribution bias exaggerates cancerspecific mortality in the screening arm and hence underestimates the mortality reduction.

A bias in the opposite direction results from the increased diagnostic testing within the screening arm and subsequent treatment that may result in death. If the fundamental role of screening, diagnostic testing or treatment for screen-detected cancer in inducing the death is not recognized and recorded appropriately, a slippery-linkage bias occurs. Slippery-linkage bias underestimates the cancer-specific mortality in the screening arm.

Both of these biases may be avoided by using all-cause mortality as an endpoint. However, this would require an extremely large cancer screening trial or an unrealistically large screening impact to show any statistical difference between the trial arms, because any specific cancer type causes of a small fraction of all deaths in the population. Despite potential biases, cancer-specific mortality is generally more informative than allcause mortality in screening trials. The biases with cancer-specific mortality can be minimized by using an independent CoD review board that is blinded to the screening status of the deceased $[8,9,13]$. However, blinding is not always complete as the members of the $\mathrm{CoD}$ committee may be able to recognize the screening status from medical records [14].

In Finland, medical doctors are obliged by law to issue a death certificate, when a person dies. That is why the coverage of death certificates is near $100 \%$. A copy of this certificate is first reviewed by forensic experts at the offices of the National Institute for Health and Welfare after which they are submitted to Statistics Finland, which maintains the official CoD registry, or returned for reissuing. Death certificates are accepted to the official registry or modified according to the WHO guidelines. An estimated $1.4 \%$ of death certificates require further clarification by the issuing medical doctor [15]. Roughly a third of the deceased undergo an autopsy in Finland [16].

In the Finnish trial, all deaths in men with diagnosed PCa were reviewed by our CoD committee in 1996-2003 and outstanding agreement $(97.7 \% ; \kappa=0.95)$ was observed between the review committee and official CoD registry. As the Finnish screening trial is very large and the number of deaths in men with PCa exceeded 2500 , all cases could not be reviewed individually. After 2003, only randomly selected deaths $(\mathrm{N}=127)$ have been reviewed by the committee.

The current study shows agreement of $94.6 \%(\kappa=0.88)$ in the SA and $95.4 \%(\kappa=0.91)$ in the CA. The agreement is slightly inferior to that previously published, but is still excellent. The Rotterdam section of the ERPSC trial reported a relatively good agreement of 90.6\% $(\kappa=0.76)$ [17]. Later, the Swedish section reported agreement of $97.3 \%(\kappa=0.94)$ in the SA and $94.7 \%(\kappa=0.89)$ in the CA [18]. The other centers of the ERSPC trial have hitherto not published separate agreement results regarding CoD ascertainment. The PLCO trial recently reported an excellent agreement between death certificates and PLCO death review committee: $97.2 \%$ in the SA $(\kappa=0.88)$ and $96.5 \%$ in the CA $(\kappa=0.87)$ [9].

A possible reason for a decline in agreement is that the trial population has become older and has accrued more comorbidities or malignancies that complicate assigning the underlying CoD. In a prospective trial, it may be beneficial in terms of cost-effectiveness to assess the comorbidity load of the deceased (especially if there are multiple malignancies present) to aid selection of cases for CoD committee review.

This study shows that the official CoD registry is likely to overestimate the role of prostate cancer in both trial arms, but slightly more $(7.4 \%)$ in the screened population than in the CA (3.1\%). Such overestimation, most likely due to the aforementioned attribution bias, dilutes the mortality reduction by screening. After correcting for this attribution bias, the HR of PCa death decreased slightly from $0.94(0.82-1.08)$ to $0.92(0.80-1.06)$.

A limitation in this study is that we have not reviewed all the deaths in men with PCa (roughly 2500) to fully ascertain the underlying causes of death. Instead, we used random sampling (altogether 442 deaths) to estimate the magnitude of 
misclassification. Possible contamination in the CA (opportunistic, non-organized PSA-testing) is likely to cause some degree of attribution bias also in the CA.

\section{Conclusions}

There appears to be a small but real differential misclassification bias in the Finnish Randomized Study of Screening for Prostate Cancer. This is probably caused by attribution bias as the screened men are more likely to be diagnosed with PCa. The PCa diagnosis can eventually be recorded as an official underlying CoD, even when the chain of events leading to death is ultimately caused by another disease. In any screening trial with disease-specific mortality as an end-point, this attribution bias should preferably be controlled with reviewing all deaths in those diseased to maximize precision, although the benefit may remain minimal.

\section{Conflict of interest}

Prof. Taari declares having received lecture fees from GlaxoSmithKline, consultant fees from Abbvie, research funding from Medivation and congress travel support from Astellas and Orion. The other authors declare no conflicts of interest.

\section{Funding}

This work was supported by the Academy of Finland; the Competitive State Research Fund (Pirkanmaa Hospital District); and Finnish Cancer Society.

\section{Authors' contribution}

Kilpeläinen: Data analysis; Manuscript writing/editing

Mäkinen: Protocol/project development; Data collection or management; Manuscript writing/editing

Karhunen: Protocol/project development; Data collection or management; Manuscript writing/editing

Aro: Protocol/project development; Data collection or management; Manuscript writing/editing

Lahtela: Protocol/project development; Data collection or management; Manuscript writing/editing

Taari: Protocol/project development; Manuscript writing/editing

Talala: Data collection or management; Manuscript writing/ editing

Tammela: Protocol/project development; Data collection or management; Manuscript writing/editing

Auvinen: Protocol/project development; Data collection or management; Data analysis; Manuscript writing/editing

\section{References}

[1] F.H. Schröder, J. Hugosson, M.J. Roobol, T.L. Tammela, M. Zappa, V. Nelen, et al. Screening and prostate cancer mortality: results of the European Randomised Study of Screening for Prostate Cancer (ERSPC) at 13 years of follow-up, Lancet 384 (2014) 2027-2035.

[2] H.B. Carter, American Urological Association (AUA) guideline on prostate cancer detection: process and rationale, BJU Int. 112 (2013) 543-547.

[3] D. Ilic, M.M. Neuberger, M. Djulbegovic, P. Dahm, Screening for prostate cancer, Cochrane Database Syst. Rev. 1 (2013) CD004720.

[4] N. Booth, P. Rissanen, T.L. Tammela, L. Määttänen, K. Taari, A. Auvinen, Healthrelated quality of life in the Finnish trial of screening for prostate cancer, Eur. Urol 65 (2014) 39-47.

[5] E.A. Heijnsdijk, T.M. de Carvalho, A. Auvinen, M. Zappa, V. Nelen, M. Kwiatkowski, et al., Cost-effectiveness of prostate cancer screening: a simulation study based on ERSPC data, J. Natl. Cancer Inst. 107 (2014) 366.

[6] T.P. Kilpeläinen, T.L. Tammela, N. Malila, M. Hakama, H. Santti, L. Määttänen, et al., Prostate cancer mortality in the Finnish randomized screening trial, J. Natl. Cancer Inst. 105 (2013) 719-725.

[7] T.P. Kilpeläinen, T.L. Tammela, N. Malila, M. Hakama, H. Santti, L. Määttänen, et al., The Finnish prostate cancer screening trial: analyses on the screening failures, Int. J. Cancer 136 (2015) 2437-2443.

[8] H.J. De Koning, J. Blom, J.W. Merkelbach, R. Raaijmakers, H. Verhaegen, P. Van Vliet, et al., Determining the cause of death in randomized screening trial(s) for prostate cancer, BJU Int. 92 (Suppl. 2) (2003) 71-78.

[9] A.B. Miller, S. Yurgalevitch, J.L. Weissfeld, Prostate, lung, colorectal and ovarian cancer screening trial project team. Death review process in the Prostate, Lung, Colorectal and Ovarian (PLCO) Cancer Screening Trial, Control. Clin. Trials 21 (Suppl. (6)) (2000) 400S-406S.

[10] T. Mäkinen, P. Karhunen, J. Aro, J. Lahtela, L. Määttänen, A. Auvinen, Assessment of causes of death in a prostate cancer screening trial, Int. J. Cancer 122 (2008) 413-417.

[11] L. Teppo, E. Pukkala, M. Lehtonen, Data quality and quality control of a population-based cancer registry. Experience in Finland, Acta Oncol. 33 (1994) 365-369.

[12] J. Cohen, A coefficient of agreement for nominal scales, Educ. Psychol. Meas. 20 (April) (1960) 37-46

[13] W.C. Black, D.A. Haggstrom, H.G. Welch, All-cause mortality in randomized trials of cancer screening, J. Natl. Cancer Inst. 94 (2002) 167-173.

[14] N.J. Williams, E.M. Hill, S.Y. Ng, R.M. Martin, C. Metcalfe, J.L. Donovan, et al., Standardisation of information submitted to an endpoint committee for cause of death assignment in a cancer screening trial - lessons learnt from CAP (Cluster randomised triAl of PSA testing for Prostate cancer), BMC Med. Res. Methodol. 15 (2015) 6.

[15] R.A. Lahti, A. Penttilä, Cause-of-death query in validation of death certification by expert panel; effects on mortality statistics in Finland, 1995, Forensic Sci. Int. 131 (2003) 113-124.

[16] R.A. Lahti, A. Penttilä, The validity of death certificates: routine validation of death certification and its effects on mortality statistics, Forensic Sci. Int. 115 (2001) 15-32.

[17] S.J. Otto, P.J. van Leeuwen, J.W. Hoekstra, J.W. Merckelbach, J.H. Blom, F.H. Schröder, et al., Blinded and uniform causes of death verification in cancer screening: a major influence on the outcome of a prostate cancer screening trial? Eur. J. Cancer 46 (2010) 3061-3067.

[18] R. Godtman, E. Holmberg, J. Stranne, J. Hugosson, High accuracy of Swedish death certificates in men participating in screening for prostate cancer: a comparative study of official death certificates with a cause of death committee using a standardized algorithm, Scand. J. Urol. Nephrol. 45 (2011) $226-232$. 